\title{
Mobilidade populacional e produção da leishmaniose tegumentar americana no Estado do Paraná, sul do Brasil
}

\section{Population mobility and production of American tegumentary leishmaniasis in the State of Paraná, southern Brazil}

\author{
Wuelton Marcelo Monteiro ${ }^{1,2}$, Herintha Coeto Neitzke-Abreu ${ }^{3}$, \\ Maria Eugenia Moreira da Costa Ferreira ${ }^{4}$, Gisely Cardoso de Melo ${ }^{1}$, \\ Maria das Graças Vale Barbosa ${ }^{1}$, Maria Valdrinez Campana Lonardoni ${ }^{5}$, \\ Thais Gomes Verzignassi Silveira ${ }^{5}$ Ueslei Teodoro 5
}

\begin{abstract}
RESUMO
São escassas as informações sobre o papel da mobilidade populacional na manutenção da leishmaniose tegumentar americana no estado do Paraná. Avalia-se a mobilidade populacional como fator de risco para esta endemia em três mesorregiões do Paraná, utilizando dados gerados na Universidade Estadual de Maringá, no período de 1987 a 2004. Foram notificados 1.933 casos, predominando os casos migrantes (54,4\%). 0s municípios com maior número de casos notificados foram Maringá (358), Doutor Camargo (108) e Terra Boa (105). Os casos rurais foram predominantemente autóctones $(89,8 \%)$, enquanto os urbanos, na maioria $(84,8 \%)$ migrantes $(\mathrm{p}<0,0001)$. Para os casos rurais autóctones, não houve predomínio entre os sexos $(\mathrm{p}=0,127)$; para os casos urbanos migrantes, prevaleceu o sexo masculino $(\mathrm{p}<0,0001)$. Os casos migrantes foram na maioria relacionados com a mobilidade intra e intermunicipal. A mobilidade populacional parece ser uma variável importante na epidemiologia desta doença no Estado do Paraná.
\end{abstract}

Palavras-chaves: Leishmaniose tegumentar americana. Mobilidade populacional. Estado do Paraná.

\section{ABSTRACT}

Information on the role of population mobility in maintaining American tegumentary leishmaniasis in the State of Paraná is scarce. Population mobility was evaluated as a risk factor for this endemic disease in three mesoregions of Paraná, using data built up at the State University of Maringá, covering 1987 to 2004 . A total of 1,933 cases were notified, mostly among migrants (54.4\%). The municipalities with the greatest numbers of cases notified were Maringá (358), Doutor Camargo (108) and Terra Boa (105). The rural cases were predominantly autochthonous (89.8\%), while the urban cases were mostly among migrants $(84.8 \%)(\mathrm{p}<0.0001)$. Among the rural autochthonous cases, there was no difference between the sexes $(\mathrm{p}=$ $0.127)$, whereas among the urban migrant cases, men predominated $(\mathrm{p}<0.0001)$. The migrant cases were mostly related to mobility within and between municipalities. Population mobility seems to be an important variable in the epidemiology of this disease in the State of Paraná.

Key-words: American tegumentary leishmaniasis. Population mobility. State of Paraná.

Até a década de 1950, a leishmaniose tegumentar americana (LTA) disseminou-se por praticamente todo o território nacional, coincidindo com o desmatamento provocado pela construção de rodovias, ferrovias e a instalação de aglomerados populacionais, com grande incidência nos Estados de São Paulo, Paraná, Minas Gerais, Ceará e Pernambuco ${ }^{3}$. Na década de 1960, a doença entrou

\footnotetext{
1. Curso de Pós-Graduação em Medicina Tropical, Universidade do Estado do Amazonas, Manaus, AM. 2. Departamento de Saúde Coletiva, Universidade Federal do Amazonas, Manaus, AM. 3. Centro de Ciências Médicas e Farmacêuticas, Universidade Estadual do Oeste do Paraná, Cascavel, PR. 4. Departamento de Geografia, Universidade Estadual de Maringá, Maringá, PR. 5. Departamento de Análises Clínicas, Universidade Estadual de Maringá, Maringá, PR.

Endereço para correspondência: Dr. Wuelton Marcelo Monteiro. Curso de Pós-Graduação em Medicina Tropical/FMTAM. Av. Pedro Teixeira 25, Dom Pedro, 69040-000 Manaus, AM.

Fax: 5592 3656-8269

e-mail: wueltonmm@ibest.com.br

Recebido para publicação em 11/05/2009

Aceito em 24/09/2009
}

em declínio, com o desmatamento já completado nas regiões mais urbanizadas do país, além da relativa estabilidade das populações rurais. Desde então, tem havido um franco crescimento da LTA, tanto em magnitude quanto em expansão geográfica em áreas de colonização antiga, em diversos estados ${ }^{415}$.

No Brasil, no período de 1980 a 2005, registraram-se 605.062 casos de LTA $^{16}$. Neste período, 13.384 casos foram notificados na Região Sul, representando 2,2 \% dos casos do Brasil, dos quais 13.206 (98,7\%) ocorreram no Estado do Paraná ${ }^{16}$. Desde o início da década de 1980, a LTA tornou-se endêmica no norte deste estado ${ }^{7022}$, atingindo proporções epidêmicas nos anos de 1993, 1994 e 1995 22, mesmo com a substituição da vegetação original de florestas pelas culturas de café, soja, milho, algodão e por pastagens, afetando indivíduos de todos os grupos etários e de ambos os $\operatorname{sexos}^{717}$.

No Estado do Paraná, atribui-se a manutenção da LTA na forma endêmica à construção de moradias próximas de encraves de 
mata, onde persiste o ciclo enzoótico da doença e à organização do espaço peridomiciliar, com condições que permitem a adaptação de flebotomíneos no ambiente antrópico ${ }^{12} 23252627$. Recentemente, foi demonstrado que uma elevada proporção dos casos de LTA diagnosticados no Estado do Paraná não se infectou no domínio domiciliar, levantando-se a hipótese de que a migração constitui-se num fator de risco para a doença, neste estado $^{17}$.

A multiplicidade de fatores que envolvem a transmissão da ITA dificulta a formulação de estratégias de controle. Neste contexto, são escassas as informações a respeito do papel da mobilidade populacional na manutenção e disseminação dessa endemia em áreas de colonização antiga do Estado do Paraná. Neste trabalho, avalia-se ainda que preliminarmente, o papel da mobilidade populacional na epidemiologia da LTA nas mesorregiões Norte Central (NC), Centro Ocidental (CO) e Noroeste (NO) do Estado do Paraná, sul do Brasil.

\section{MATERIAL E MÉTODOS}

Área de estudo. As mesorregiões $\mathrm{NC}, \mathrm{CO}$ e $\mathrm{NO}$ do Estado do Paraná (Figura 1) situam-se entre os meridianos de $51^{\circ} 30^{\prime}$ e $54^{\circ} 00^{\prime}$ longitude Oeste e os paralelos de $22^{\circ} 30^{\prime}$ e $24^{\circ} 35^{\prime}$ latitude Sul, abrangendo 162 municípios, numa área de $61.250,8 \mathrm{~km}^{2} \mathrm{e}$ uma população de 2.816 .800 habitantes, com 2.364 .414 (83,9\%) na zona urbana e $452.386(16,1 \%)$ na zona rural ${ }^{6}$. A maioria dos municípios dessas mesorregiões está classificada no nível médio inferior de desenvolvimento humano, sendo o setor primário o mais importante na participação setorial do valor adicionado fiscal. Porém, destacam-se as regiões metropolitanas de Maringá e de Londrina, bem como cidades como Umuarama e Cianorte, com Índice de Desenvolvimento Humano elevado. A agropecuária extensiva contribui com a maior parcela do valor bruto da produção silvo-agropecuária. A maioria dos municípios teve taxas negativas de crescimento da população total, de 1970 a 2000, e o crescimento geométrico foi negativo para a população rural, porém positivo para a população urbana, de 1991 a 2000. Atualmente, a população da área rural representa, em alguns municípios, menos de $20 \%$ da população total ${ }^{6}$.

As mesorregiões supracitadas foram ocupadas na primeira metade do século XX, constituindo zonas agrícolas pioneiras do Brasil $^{10}$. 0 desmatamento para a ocupação do solo, especialmente intenso nas áreas das mesorregiões referidas, foi preponderante para o desaparecimento da cobertura vegetal originária, restando apenas algumas áreas testemunhas ou associadas à presença de cursos de água. A colonização, a construção de rodovias e ferrovias e, em especial a atividade agrícola, foram os responsáveis pela transformação acelerada da paisagem.

O clima nas mesorregiões NC, CO e NO é do tipo tropical de transição para subtropical, com temperaturas médias anuais entre 20 e $22^{\circ} \mathrm{C}$, médias do mês mais quente superior a $22^{\circ} \mathrm{C}$ e invernos brandos com períodos de seca hibernal pouco pronunciada, dos meses de julho a setembro. A precipitação anual avaliada por um período de cinco anos indica média de $1.600 \mathrm{~mm} / \mathrm{ano}$, sendo novembro o mês mais chuvoso e agosto o mais seco?.

Procedimentos. Os dados utilizados para a realização deste trabalho foram gerados no período de 1987 a 2004, no Laboratório de Ensino e Pesquisa em Análises Clínicas da Universidade Estadual de Maringá (LEPAC/UEM), onde foram feitos os diagnósticos de LTA. Os pacientes foram considerados positivos
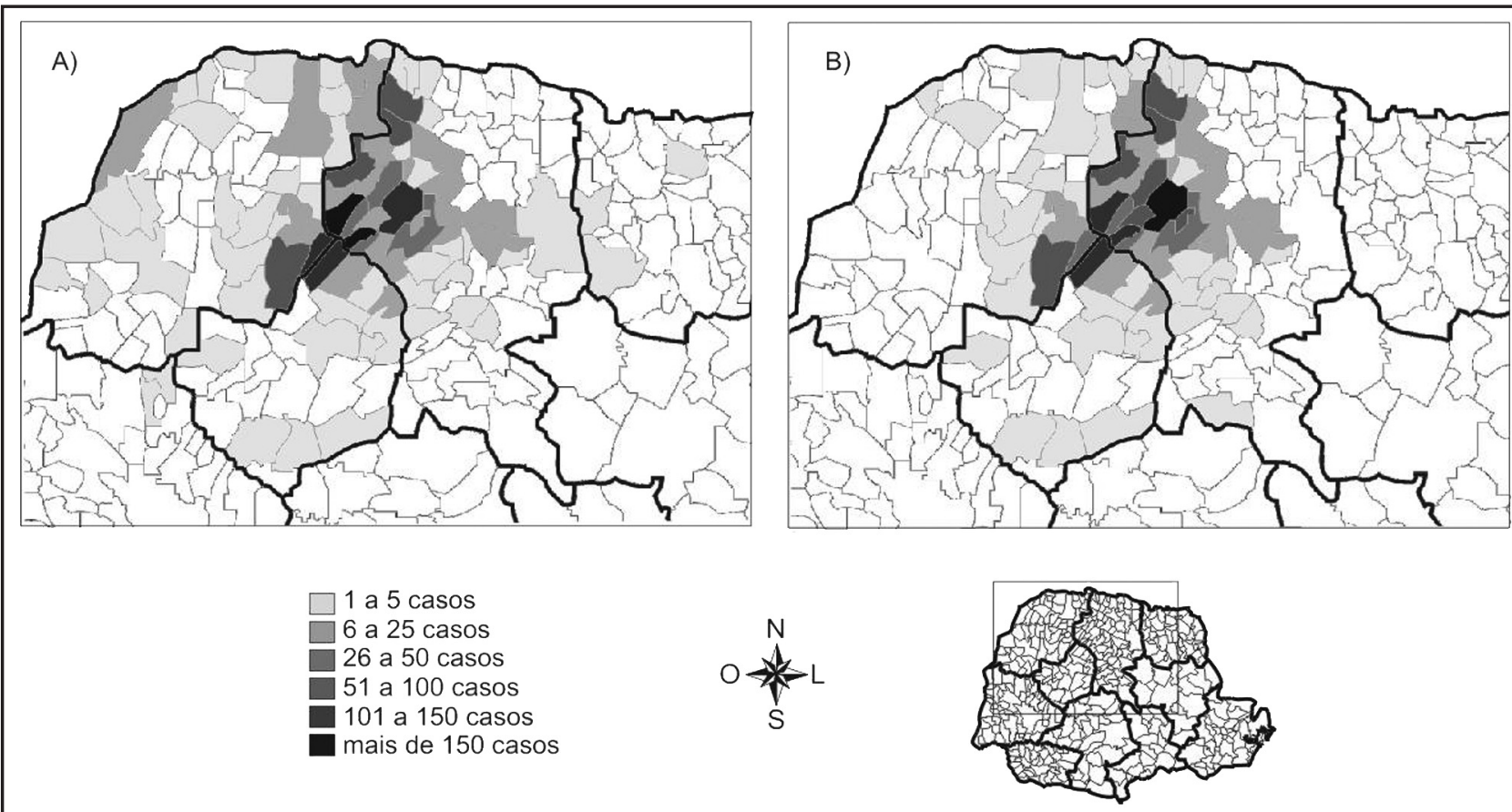

\section{FIGURA 1}

Distribuição espacial dos casos de leishmaniose tegumentar americana diagnosticados no Laboratório de Ensino e Pesquisa em Análises Clínicas da Universidade Estadual de Maringá, de 1987 a 2004, conforme município de infecção (A) e notificação (B). 
quando tiveram pelo menos um dos seguintes testes diagnósticos positivo: pesquisa direta de Leishmania, imunofluorescência indireta (título $\geq 40$ ) ou reação intradérmica de Montenegro (diâmetro $\geq 5 \mathrm{~mm}$ ). Os dados coletados dos pacientes foram: município de moradia, município de infecção, zona (rural/ urbana) de moradia e infecção, ocupação, idade e sexo. Foram analisados apenas os casos notificados nos municípios inseridos nas mesorregiões NC, CO e NO do Paraná.

Neste trabalho, o conceito de migrante corresponde à categoria de migrante de retorno ${ }^{13}$, representada por indivíduos que empreendem múltiplas ou repetidas migrações, retornando eventualmente ao ponto de origem, mas não de forma definitiva; este migrante não se fixa definitivamente na origem nem no destino, pois a migração representa um ponto de contato permanente entre um e outro. Conforme Zaba e Clarque ${ }^{29}$, este tipo de migração, em seu sentido mais amplo, inclui processos tais como a urbanização, o turismo e a migração pendular. 0 entendimento desses conceitos deve levar em consideração as oportunidades de trabalho e as estratégias para assegurar a reprodução de suas condições de existência, as quais podem mudar radicalmente a relação entre população nativa ou residente e seu meio ambiente.

Conforme o provável local de infecção, os casos foram divididos em duas categorias: 1) autóctones: indivíduos que provavelmente adquiriram a infecção no domicílio, no peridomicílio e, nos casos rurais, na propriedade rural onde reside e, 2) alóctones ou migrantes: indivíduos que provavelmente se infectaram fora do domínio doméstico ou do seu entorno. Os casos alóctones, conforme o tipo de deslocamento, foram reclassificados em três grupos: a) casos em migrantes relacionados aos deslocamentos intramunicipais; b) casos em migrantes relacionados com os deslocamentos intermunicipais; c) casos em migrantes relacionados com os deslocamentos interestaduais ou internacionais.

A análise estatística foi realizada pelo teste do qui-quadrado, a nível de 5\% de significância, utilizando-se o programa Epi-Info versão 3.4.3.

\section{RESULTADOS}

No período de 1987 a 2004, foram notificados 1.933 casos de LTA. Destes, para 1.588 (81,9\%), provenientes de 74 municípios, foi possível identificar o município onde ocorreu a infecção (Figura 1). Os casos migrantes representaram 54,4\% (863) e os casos autóctones 45,6\% (725).

Verificou-se que $648(40,8 \%)$ indivíduos moravam na zona rural e $940(59,2 \%)$ na urbana. Entre os casos rurais, $582(89,8 \%)$ eram autóctones e $66(10,2 \%)$ eram migrantes, enquanto entre os moradores urbanos, 143 (15,2\%) eram autóctones e $797(84,8 \%)$ eram migrantes $(\mathrm{p}<0,0001)$. Para os casos rurais autóctones observaram-se 322 (55,3\%) indivíduos do sexo masculino e 260 (44,7\%) do sexo feminino, enquanto entre os casos rurais migrantes observaram-se $43(65,2 \%)$ do sexo masculino e $23(34,8 \%)$ do sexo feminino, não havendo diferença estatística entre o número de homens e mulheres para este grupo $(\mathrm{p}=0,127)$. Para os casos urbanos autóctones, observaram-se $67(46,9 \%)$ indivíduos do sexo masculino e $76(53,1 \%)$ do sexo feminino, enquanto entre os casos urbanos migrantes observaram-se $715(89,7 \%)$ do sexo masculino e $82(10,3 \%)$ do sexo feminino, havendo predomínio significativo do sexo masculino para este grupo $(\mathrm{p}<0,0001)$.

Os municípios com maior número de casos notificados foram Maringá (358 casos), Doutor Camargo (108), Terra Boa (105), São Jorge do Ivaí (104), Colorado (80), Sarandi (76), Cianorte (69), Nova Esperança (63), Lobato (61) e Mandaguaçu (60) (Figura 1). A autoctonia dos casos predominou em 38 municípios, enquanto em 32 municípios predominaram os casos entre migrantes. Em quatro municípios, o número de casos autóctones foi igual ao número de migrantes. Destacaram-se pelo número de casos migrantes os municípios de Maringá (74,6\%; 267 migrantes), Sarandi (89,5\%; 68 migrantes), São Jorge do Ivaí (54,8\%; 57 migrantes), Paiçandu (94,8\%; 55 migrantes), Doutor Camargo (48,1\%; 52 migrantes), Cianorte (49,3\%; 34 migrantes), Colorado (57,5\%; 34 migrantes), Nova Esperança $(52,4 \% ; 33$ casos), Mandaguaçu (43,3\%; 23 casos), Terra Boa (21,9\%, 23 casos), Lobato (36,1\%; 22 casos) e Ivatuba (70\%; 21 migrantes).

No geral, destacaram-se pela evasão de casos os municípios de Jussara (94 casos), São Jorge do Ivaí (50), Terra Boa (49), Maringá (16), Doutor Camargo (14), Lobato (13) e Colorado (12). Os municípios de Maringá (211 casos), Sarandi (58), Paiçandu (42), Mandaguaçu (19), Nova Esperança (18) e Marialva (13) destacaram-se principalmente como centros receptores. Nos municípios de São Jorge do Ivaí, Doutor Camargo, Colorado, Terra Boa, Lobato e Ivatuba predominaram os casos relacionados com a mobilidade intramunicipal. Em Maringá, Sarandi, Paiçandu, Cianorte, Nova Esperança e Mandaguaçu predominaram os casos intermunicipais.

Os casos interestaduais originaram-se de Mato Grosso (59), São Paulo (23), Rondônia (22), Mato Grosso do Sul (20), Bahia (4), Minas Gerais (4), Pará (2), Roraima (2), Santa Catarina (2), Acre (1), Amazonas (1) e Tocantins (1), bem como o Paraguai (9). Na Tabela 2, nota-se que a maioria dos casos interestaduais foram registrados em Maringá (85), Sarandi (9), Nova Esperança (4), Paiçandu (3), Doutor Camargo (2) e Mandaguaçu (2). Em Maringá observaram-se nove casos provenientes do Paraguai.

A distribuição por faixas etárias mostrou diferenças significativas entre os grupos de casos autóctones e migrantes $(\mathrm{p}<0,0001)$.Verificou-se que entre os 794 casos migrantes, as faixas etárias mais acometidas foram entre 30 e 64 anos (66,6\%) e entre 15 e 29 anos (23,4\%). Registraram-se 13 (1,6\%) casos em menores de 14 anos e não foram observados casos em menores de cinco anos. Entre os 667 casos entre não-migrantes, as faixas etárias mais acometidas também foram aquelas entre 30 e 64 anos (51,4\%) e entre 15 e 29 anos (27,3\%). No entanto, observou-se maior proporção de casos em menores de 14 anos (13,2\%) e 0 acometimento de menores de cinco anos (2,7\% dos casos).

$\mathrm{Na}$ Tabela 1, verifica-se que as ocupações mais freqüentes para os casos migrantes foram as ocupações do setor primário, com 235 (29,6\%) casos; os autônomos do setor terciário, com $189(23,8 \%)$ casos e os empregados do setor terciário, 


\section{TABELA 1}

Ocupações dos casos de leishmaniose tegumentar americana diagnosticados no Laboratório de Ensino e Pesquisa em Análises Clínicas da Universidade Estadual de Maringá, de 1987 a 2004

\begin{tabular}{lrrrrrrr}
\hline & \multicolumn{2}{c}{ Migrantes } & & \multicolumn{2}{c}{ Não migrantes } \\
\cline { 2 - 3 } Ocupação & $\mathrm{n}^{0}$ & $\%$ & & $\mathrm{n}^{0}$ & $\%$ & Total \\
\hline Ocupações do setor primário & 235 & 29,6 & & 322 & 48,3 & 557 \\
Donas de casa & 40 & 5,0 & & 170 & 25,5 & 210 \\
Autônomos do setor terciário & 189 & 23,8 & & 1 & 0,1 & 190 \\
População infantil e estudantes & 44 & 5,6 & & 97 & 14,5 & 141 \\
Aposentados & 77 & 9,7 & & 62 & 9,3 & 139 \\
Empregados do setor terciário & 108 & 13,6 & & 2 & 0,3 & 110 \\
Autônomos do setor secundário & 70 & 8,8 & & 3 & 0,5 & 73 \\
Desempregados & 21 & 2,7 & & 0 & 0,0 & 21 \\
Empregados do setor secundário & 9 & 1,1 & & 0,3 & 11 \\
Outros & 1 & 0,1 & & 8 & 1,2 & 9 \\
\hline Total & 794 & 100,0 & 667 & 100,0 & 1.461 \\
\hline
\end{tabular}

Setor primário: atividades agrícolas, mineração, pesca, pecuária, extrativismo vegetal e caça; setor secundário: setor da economia que transforma as matériasprimas em produtos industrializados; setor terciário: comércio, educação, saúde, telecomunicações, serviços de informática, seguros, transporte, serviços de limpeza, alimentação, turismo, bancários, administrativos e de transportes.

com 108 (13,6\%) casos. Para esta categoria, os autônomos dos setores terciário e secundário somaram $259(32,6 \%)$ casos. Entre os casos não migrantes, predominaram as ocupações do setor primário, com 322 (48,3\%) casos; as donas de casas, com $170(25,5 \%)$ casos; a população infantil e os estudantes, com 97 (14,5\%) casos; e os aposentados, com 62 (9,3\%) casos (Tabela 1).

\section{DISCUSSÃo}

0 número de casos de LTA no Brasil vem aumentando, particularmente a partir de 1985, com destaque para as regiões Nordeste e Norte, onde ocorre a maioria das notificações ${ }^{15}$. Embora o elevado número de indivíduos infectados existentes no país não traduza uma quantidade igual de formas graves da doença $^{11123}$, chama a atenção o caráter francamente expansivo da endemia ao longo do território nacional ${ }^{341516}$. Os casos registrados no Estado do Paraná, a partir de 1980, mostram 0 perfil endêmico da doença ${ }^{71720}$, sobretudo no norte e oeste deste estado ${ }^{2717}$.

Verificou-se a ocorrência da doença em 74 municípios das mesorregiões NC, CO e NO do Estado do Paraná, confirmando a ampla distribuição desta endemia nessas mesorregiões ${ }^{71720}$. Estes municípios pertencem ao circuito Paranapanema de produção de $\mathrm{LTA}^{2}$, recentemente, denominado circuito Paraná-Paranapanema ${ }^{18}$, com duas áreas de grande intensidade de casos. Na primeira área, na bacia do rio Pirapó, destacam-se os municípios de Lobato e Colorado e na segunda, na bacia do rio Ivaí, os municípios de São Jorge do Ivaí, Terra Boa, Jussara, Cianorte e Doutor Camargo. 0 predomínio de casos migrantes em 32 dos 74 municípios, onde foram registrados casos de LTA, reforça a importância da mobilidade populacional na epidemiologia desta doença no Estado do Paraná. Trinta e nove municípios apresentaram no máximo cinco casos e nestes também predominaram os casos entre os migrantes.

Os municípios de Jussara e Terra Boa apresentaram elevados índices de autoctonia. Aparentemente, a colonização desses municípios trouxe contingente populacional humano mais estável que fixou suas residências nas áreas rurais, enquanto os limites ecológicos dos focos de LTA se restringem às matas residuais. As características ambientais destas localidades rurais favorecem a infecção por Leishmania, pois é muito comum nestas a proximidade de domicílios e anexos (galinheiros, chiqueiros, paióis e outros) das matas remanescentes, onde persistem focos enzoóticos de Leishmania 72202223252627 .

Nos municípios de São Jorge do Ivaí, Nova Esperança, Mandaguaçu, Colorado, Lobato, Ivatuba e Ourizona há um contingente significativo de casos entre moradores da zona urbana, que se infectaram na área rural dos próprios municípios. Para estes casos, o trabalho agrícola volante desenvolvido pelos moradores das cidades contribuiu para o registro. Atualmente, muitos antigos ocupantes da zona rural migraram para a sede dos municípios, continuando as atividades no campo, pois a baixa qualificação profissional dificulta a absorção dos mesmos pelo restrito mercado de trabalho urbano.

Destacaram-se pela autoctonia dos casos urbanos os municípios de Maringá e Cianorte. 0 registro de casos urbanos autóctones da zona urbana ou periurbana destes municípios já foi relatado anteriormente ${ }^{1720}$, sendo que a produção dos casos nestes focos vem sendo creditada ao modelo de ocupação do espaço $^{7172124}$, em grande parte por antigos moradores de zonas rurais. Os hábitos culturais comuns entre as populações rurais, como a produção e/ou estocagem de gêneros alimentícios de subsistência na periferia das cidades, podem criar um ambiente favorável à circulação de reservatórios de Leishmania e à procriação de flebotomíneos, uma vez que estes hábitos propiciam 0 acúmulo de matéria orgânica e a umidade do solo.

Apesar do registro de casos provenientes de outros estados ou do Paraguai, a grande maioria dos casos está relacionada com a mobilidade intramunicipal e intermunicipal. De fato, especialistas apontam para a situação de migrações de curta distância, predominantemente intrarregionais, podendo ser sazonais em áreas de modernização agrícola ou intermunicipais em áreas de maior urbanização ${ }^{14}$, onde se insere o norte do Estado do Paraná. Assim, a maior densidade da rede urbana tenderá a produzir fluxos de curta distância. No caso de Maringá e dos municípios vizinhos, observou-se importante evasão de casos de LTA a partir dos municípios das bacias dos rios Ivaí e Pirapó e, em escala menos significativa, de outros estados, destacando-se os pertencentes à Amazônia Legal.

A grande proporção de casos envolvendo jovens e mulheres que, provavelmente, adquiriram a infecção no peridomicílio e domicílio, sugere a existência do ciclo de transmissão domiciliar e peridomiciliar nessas mesorregiões do Estado do Paraná. Apesar destes casos apresentarem, na grande maioria, moradia permanente na zona rural, a vulnerabilidade não parece estar ligada ao trabalho agrícola, já que a proporção de pessoas ocupadas com atividades domésticas foi semelhante àquela das pessoas envolvidas com o trabalho rural. 
A concentração dos casos alóctones nas faixas etárias produtivas, a baixa incidência na população infantil, a inexistência de casos em menores de cinco anos e o predomínio do sexo masculino evidenciam o perfil ocupacional destes casos de LTA. Como a maioria dos indivíduos reside na zona urbana, mas adquiriu a infecção na zona rural, é muito provável que o trabalho agrícola volante, de característica rotativa e informal, desenvolvido pelos moradores das cidades, tenha contribuído para o grande número de casos de LTA em moradores da zona urbana.

A migração é um fator que aumenta a vulnerabilidade da população, por prejudicar o acesso a bens de consumo e aos serviços públicos ${ }^{5}$, como os serviços de saúde. No Estado do Paraná, verificou-se que aspectos importantes que envolvem 0 acompanhamento dos pacientes com LTA, como o exame clínico, 0 registro em prontuário, o acompanhamento e a avaliação final dos pacientes e 0 uso de medidas de prevenção deixaram de ser realizados durante 0 atendimento ${ }^{81928}$. Não se sabe até que ponto a mobilidade populacional pode ter contribuído para estas deficiências no atendimento, mas é possível que a população migrante se torne vulnerável, pela impossibilidade do acompanhamento deste grupo pelas equipes de saúde, pela falta de conhecimento do novo lugar e das fracas ou inexistentes redes sociais, colocando maiores cargas ambientais sobre os migrantes.

É possível que o número de casos de LTA apresentados estejam subestimados, em face de que apenas uma parcela da população poderia ter buscado atendimento no LEPAC/UEM no período do estudo. Porém, deve-se considerar que os resultados deste trabalho são mais precisos, em virtude das falhas no processo de notificação e devido ao diagnóstico mais preciso da doença e maior capacidade de obtenção de dados epidemiológicos em áreas que servem de base para pesquisas nas mesorregiões em pauta ${ }^{7820}$.

As migrações humanas possivelmente desempenham papel importante na difusão e na exacerbação da LTA no Paraná, envolvendo muitos trabalhadores agrícolas volantes, que por não possuírem, de início, experiência com a LTA, não adotam medidas de proteção com relação aos vetores e ao manejo ambiental. Constata-se ainda que zonas com alta transmissão são capazes de influenciar a epidemiologia da doença em áreas extensas, justificando-se a concentração dos esforços para reduzir a importância de tais áreas identificadas pelos trabalhos de espacialização.

\section{AGRADECIMENTOS}

Aos responsáveis pelo Laboratório de Ensino e Pesquisa em Análises Clínicas da Universidade Estadual de Maringá, por permitirem e facilitarem 0 acesso aos dados utilizados neste estudo.

\section{REFERÊNCIAS}

1. Castro EA, Thomaz-Soccol V, Membrive N, Luz E. Estudo das características epidemiológicas e clínicas de 332 casos de leishmaniose tegumentar notificados na região norte do Estado do Paraná de 1993 a 1998. Revista da Sociedade Brasileira de Medicina Tropical 35: 445-452, 2002.
2. Fundação Nacional de Saúde. Vigilância e monitoramento da leishmaniose tegumentar americana em unidades territoriais-Brasil, 1994-2001. http://funasa. gov.br (acessado em 05/Fev/2005).

3. Furtado TA. Leishmaniose tegumentar americana. In: Machado-Pinto J (ed) Doenças infecciosas com manifestações dermatológicas. Editora Medsi, Rio de Janeiro, p. 319-336, 1994.

4. Furtado TA, Vieira JBF. Geografia da leishmaniose tegumentar americana no Brasil. Anais Brasileiros de Dermatologia 57:135-140, 1982.

5. Hogan DJ Mobilidade populacional, sustentabilidade ambiental e vulnerabilidade social. Revista Brasileira de Estudos de População 22:323-338, 2005.

6. Instituto Paranaense de Desenvolvimento Econômico e Social. Indicadores e mapas temáticos para o planejamento urbano e regional. Ipardes, Curitiba, 2003.

7. Lima AP, Minelli L, Comunello E, Teodoro U. Distribuição da leishmaniose tegumentar por imagens de sensoreamento remoto orbital, no Estado do Paraná, Sul do Brasil. Anais Brasileiros de Dermatologia 77:681-692, 2002.

8. Lima MVN, Oliveira RZ, Lima AP, Felix MLO, Silveira TGV, Rossi RM, Teodoro U Atendimento de pacientes com leishmaniose tegumentar americana: avaliação nos serviços de saúde de municípios do noroeste do Estado do Paraná, Brasil. Cadernos de Saúde Pública 23:2938-2948, 2007.

9. Maack R. Geografia física do Estado do Paraná. Editora José Olímpio, Rio de Janeiro, 1981.

10. Machado LO. A fronteira agrícola no Brasil. In: Becker B, Christofoletti A, Dadidivich FR, Geiger PP (eds) Geografia e meio ambiente no Brasil. Editora Hucitec, São Paulo, p. 35-53, 1995.

11. Marsden PD. Mucosal leishmaniasis (Espundia Escomel, 1911). Transactions of the Royal Society of Tropical Medicine and Hygiene 80:859-876, 1986.

12. Membrive NA, Rodrigues G, Membrive U, Monteiro WM, Neitzke HC, Lonardoni MVC, Silveira TGV, Teodoro U. Flebotomíneos de municípios do Norte do Estado do Paraná, sul do Brasil. Entomología y Vectores 11:673-680, 2004.

13. Menezes MA. Questionamentos às categorias "migrante de retorno" e "migrante". In: Anais da I Conferência sobre "A Questão Regional e os Movimentos Sociais no Terceiro Mundo”. Associação de Geografia Teorética, Rio Claro, p. 44, 1992.

14. Menezes MLP. Tendências atuais das migrações internas no Brasil. http://www. ub.es/geocrit/sn-69-45.htm (acessado em 17/03/2009).

15. Ministério da Saúde. Manual de controle da leishmaniose tegumentar americana.

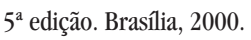

16. Ministério da Saúde. Leishmaniose Tegumentar Americana - Distribuição de casos confirmados de LTA de 1980 a 2005. http://dtr2001.saude.gov.br/svsepi/ situacao_doencas/planilhas_doencas.htm (acessado em 12/12/2006).

17. Monteiro WM, Neitzke HC, Lonardoni MVC, Silveira TGV, Ferreira MEMC, Teodoro U. Distribuição geográfica e características epidemiológicas da leishmaniose tegumentar americana em áreas de colonização antiga do Estado do Paraná, Sul do Brasil. Cadernos de Saúde Pública 24:1291-1303, 2008.

18. Monteiro WM, Neitzke HC, Lonardoni MVC, Silveira TGV, Teodoro U, Ferreira MEMC. Pólos de produção de leishmaniose tegumentar americana no norte do estado do Paraná, Brasil. Cadernos de Saúde Pública 25:1083-1092, 2009.

19. Roberto ACBS, Misuta NM, Nerilo-Sobrinho A, Lima AP, Silveira TGV, Fukushigue Y, Peixoto PR, Ferreira MEMC, Teodoro U. Avaliação da terapia com antimoniato de N-metil glucamina e de notificação de leishmaniose tegumentar. Anais Brasileiros de Dermatologia 72:129-136, 1997.

20. Silveira TGV, Arraes SMAA, Bertolini DA, Teodoro U, Lonardoni MVC, Roberto ACBS, Ramos M, Nerilo-Sobrinho A, Ishikawa EA. Observações sobre o diagnóstico laboratorial e a epidemiologia da leishmaniose tegumentar no estado do Paraná, sul do Brasil. Revista da Sociedade Brasileira de Medicina Tropical 32:413-423, 1999.

21. Silveira TGV, Roberto ACBS, Zanzarini PD, Venazzi EAS, Mangabeira HN, Teodoro U, Lonardoni MVC, Arraes SMAA. Leishmaniose tegumentar americana: surto na região urbana, no município de Maringá, Norte do Paraná. Revista da Sociedade Brasileira de Medicina Tropical 37 (supl III): 49, 2004.

22. Silveira TGV, Teodoro U, Lonardoni MVC, Guilherme AL, Toledo MJO, Ramos M, Arraes SMAA, Bertolini DA, Spinosa RP, Barbosa 0. Aspectos epidemiológicos da leishmaniose tegumentar em área endêmica do Estado do Paraná, Brasil. Cadernos de Saúde Pública 12:141-147, 1996. 
23. Teodoro U, Balduíno J, Thomaz-Soccol V, Barbosa OC, Ferreira MEMC, Lozovei AL, Silveira TGV, Roberto ACBS. Environmental sanitation and peridomiciliar organisation as auxiliary practices for the control of phlebotomines in Paraná State, Southern Brazil. Brazilian Archives of Biology and Technology 42:307-314, 1999.

24. Teodoro U, Kühl JB, Santos DR, Rodríguez M, Santos ES, Maróstica LM. Flebotomíneos coletados em florestas remanescentes e abrigos de animais silvestres de zoológico no perímetro urbano de Maringá, Sul do Brasil. Estudo Preliminar. Revista da Sociedade Brasileira de Medicina Tropical 31:517-522, 1998.

25. Teodoro U, Santos DR, Santos AR, Oliveira O, Poiani LP, Silva AM, Neitzke HC, Monteiro WM, Rossi RM, Lonardoni MVC, Silveira TGV. Informações preliminares sobre flebotomíneos do norte do Paraná. Revista de Saúde Pública 40:327-330, 2006.

26. Teodoro U, Silveira TGV, Santos AR, Santos DR, Santos ES, Oliveira O, Kühl JB. Frequiência da fauna de flebotomíneos no domicílio e em abrigos de animais domésticos no peridomicílio, nos municípios de Cianorte e Doutor Camargo, Estado do Paraná, Brasil. Revista de Patologia Tropical 30:209-224, 2001.

27. Teodoro U, Silveira TGV, Santos DR, Santos ES, Santos AR, Oliveira O, Kühl JB, Alberton D. Influência da reorganização, da limpeza e da desinsetização de edificações na densidade populacional de flebotomíneos, no município de Doutor Camargo, Estado do Paraná, Brasil. Cadernos de Saúde Pública 19:1801-1813, 2003.

28. Teodoro U, Spinoza RP, La Salvia Filho V, Guilherme ALF, Lima AP, Junqueira GMB, Misuta NM, Nerilo-Sobrinho A, Lima EM. Da necessidade de se adotar e divulgar esquemas terapêuticos para tratamento de leishmaniose tegumentar no Paraná. Revista da Sociedade Brasileira de Medicina Tropical 33:199-204, 1991.

29. Zaba B, Clarke JI. Environment and population change. Ordina Editions, Liège, 1994. 